\title{
Namen und Sachen.
}

\section{. Von Georg Hoffmann.}

I. Mene, mene tekel upharsin.

Herr Clermont-Ganneau hat im Journ. asiat. t. VIII p. 36, I 886 zwar den Schlüssel zum Verständniss von Daniel $\mathrm{V}, 25 \mathrm{ff}$. dargereicht, aber wie $\mathrm{T}_{\mathrm{H}}$. NöLdEkE bei Besprechung seiner Arbeit in dieser Zeitschrift I886, p. 414 ff. mit Recht meint, dasselbe nicht erschlossen. Die Lösung des Räthsels will die folgende Deutung versuchen.

Daniel V, 25. So lautet die angeschriebene Schrift: "Eine Mine, eine Mine in Sekel, und (zwei) Halb. minen". 26. Dies ist die Deutung der Worte: "Eine Mine": Gott hatte dein Reich voll ausgezählt. 27.,"Sekel": Gewogen bist du auf.der Wagschale und mangelhaft befunden. 28. "Halbmine": Zerbrochen ist dein Reich worden und dem Meder und Perser gegeben.

I. פרסין •3.

I. מנה ist die rechnungsmässige (leichte) Mine nach ihrem Nominalwerthe, also die vollwichtige dem Löwen von Abydos; vgl. ZDMG 21, 467. Gott hatte dem Belsazar sein Reich vollständig übergeben d. h. so blühend wie Nebukadnezar es hinterlassen (4, 33 vgl. 5, 22), mithin die Mine vollwichtig ausgezahlt.

2. Apposition ist - die Mine in Sekelstücken d. h. Dariken oder Goldstateren. Madjden, Fervish Coinage 1864 p. 274 (und sonst) sagt: 'ancient coins are always struck below their nominal weight'. Durch 
Nachwiegen ermittelte man, wie weit die Geldstücke hinter dem Normalgewicht חסיר waren; s. F. Hultsch Gr. u. Rö. Metrol. 1882, \$ 42, 14. - In Vers 27 ist תקל allein statt wiederholt, weil die Auslegung sich auf jenes Wort zuspitzt; ebenso steht Vers 28 פרס mit Rücksicht auf „Persien“ statt. פ"פ.

3. פרסיץ sind dem Sinne nach sicher $z$ wei Halbminen: das beweist die Deutung auf Teilung zwischen dem Meder (Darius) und Perser (Cyrus). Man hat den Dualis nur nicht gesprochen, weil er in diesem Falle mehr als in بִ Dan.

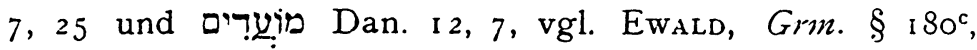
gegen die Sprachanalogie ist, wie Nöldeke p. 416 sehr fein bemerkt hat. Nichtsdestoweniger bed e u t e $t$ in allen diesen Fällen das Wort im Plural den Dualis, grade wie Gen. 25,$24 ; 38,27$.

Aus dem Zusaze תקל geht hervor, dass die Mine und Halbminen als Geldgewichte gemeint sind, wie laut seiner von GEIGER trotz DE VOGÜE's Widerspruch richtig gedeuteten Inschrift, das persische Talent von Abydos. ${ }^{\mathrm{I}}$ ) Es liegt auf der Hand, welch passendes Bild der Königswürde sie darstellen, da die Prägung des Goldsekels, des Dareikos, Vorrecht des Reichsoberhauptes blieb.

Bei der Besprechung der Wurzeln NöldEKE p. 416 mit gewohnter Meisterschaft führt, ist nur

1) DE VoGüÉ, Mélanges d'archéol. orient. p. 183. Zur Erleichterung

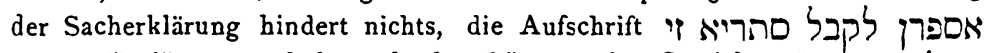
NEDZ für jünger zu halten als den Löwen; das Gewicht wäre aus einem alten persischen Goldstücktalent $(25,20 \mathrm{Klgr}$ ) zur Zeit der Inschrift zu einem attischen Silbertalent $(26,196 \mathrm{Klgr}$.) adjustiert, vgl. HuLTSCH, Griech. 2. Rö. Metrol. $\$ 46$, 12. 15. BRandis p. 54. Man darf hinter אריא allerdings keine Ziffer erwarten; da es dann סר ס heissen müsste. Man hat zu übersezen: „vollständig im Vergleich zu den (d. h. seinen, d. i. des Talents, rechnungsmässig zugehörigen) Silberstateren". GARDE, Armcnische Studien 2040. Zu $=\sum \tau \dot{c} \tau \varepsilon \_\alpha \alpha$, Gemahlinn Artaxerxes' II.; auch Name einer Frau und einer Tochter Darius' III. 
seine Meinung auffällig, dass „,gespaltene Hufe haben“, statt „Hufe haben“; das erstere

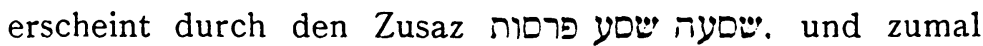
Deut. 14, 6 וישסעה שסע שתי פרסות gradezu ausgeschlossen. Lev. I I, 4, an welcher Stelle allein Gesenrius im Thes. die Deutung.,Hufe spalten" anerkennt, liegt indessen ein Schreiberversehn vor. Hinter פרסה ist das gefallen, welches Deut. I 4,7 darbietet. In dem מפריסי הזרסה השסועה könnte das Spalten doch wohl nicht zweimal ausgedrückt sein. Wenn nun auch an diesen Stellen keine Beziehung auf ein Etymon von ist die Ableitung des gebrochenen (zunächst thierischen) Fussknochens von פרס desswegen wahrscheinlich, weil dieser Wortstamm insbesondere auch vom Knochenzerbrechen gebraucht wird: Mi. 3, 3, vgl. בית המרת in LEvy's Wb. zum Talmud, und bei Nöldeke p. 418.

Nöldeke's Ansicht, dass auf dem Löwengewicht פרש „Halbmine" assyrischer Aussprache ist, also im Aramäischen Fremdwort, lässt sich damit bestätigen, dass für פ die Bedeutung „Hälfte“ nur in פרס als „Halbmine" nachgewiesen ist, während sonst das Wort wie die Wurzel nur auf „Stück, Bruchteil“" geht. Daher es auch gerathener ist, an der Danielstelle פריסת nicht mit ,halbiert worden ist" zu übersezen. Wie nahe es lag, פרס פר (פר ) auf den $\mathrm{Bruch}$ einer Mine in Barren (Metallkuchen) anzuwenden,

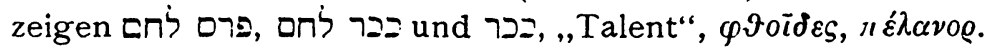

NöLDEKE macht auf die Dialektmischung der Gewichtlöwen aufmerksam. Mich dünkt sie erklärlich genug, wenn man beachtet, dass die phöniz. Schrift auf einem Teil derselben denselben Inhalt doppelt in aramäischer und in phönizischer Sprache schreibt. Z. B. steht nach $V_{\text {AUX }}$ und

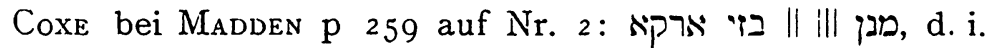
aramäisch; dann, mit Voranstellung der Zahlen, משראו

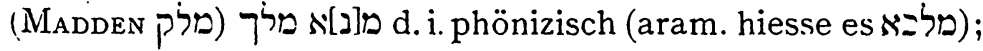
endlich ähnliches auf assyrisch. Auf einigen Gewichten (p. 26I) ist die Inschrift nur phönizisch, sogar in der 
Orthographie מנא statt. Interessant ist dabei, dass die Bezeichnung „nach dem Landesgewicht" aramäisch ist, also zeigt, dass die breite Masse der assyrischen Unterthanen dieser Sprache war, während das Assyrische und Babylonische als Volkssprache wohl nur ein sehr beschränktes Gebiet inne hatte. Dieselben Gewichte heissen „des Königs“ für die phönizischen Kautleute. Da dem kgl. Dolmetscher nun wohl die aramäische Sprache geläufiger war als die phönizische, so mischte er nicht nur die Orthographie sondern auch die Sprache; Manden

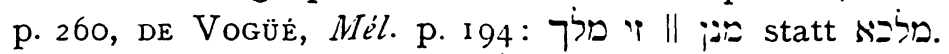

Auch die Schreibung שקלן, wie neuerdings wieder Clervont-Ganneau a. a. O. p. 45 liest, mit könnte man der Rücksicht auf die phön. Händler zuschreiben; DE VoGüÉ, Mél. p. 187 las ausdrücklich Doch ist auch nicht ausgeschlossen, dass die Schreibung ip ebensogut aramäisch war, wie die von $r$. Denn sowohl im Hebr. wie dem älteren Aram. sind i $\forall y$ wahrscheinlich polyphone Buchstaben gewesen; i mochte ähnlich wie $j$ einen Laut 2 wischen $i$ und $\urcorner$, und $*$ einen

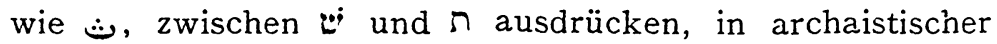
Orthographie. Eher עמערת סמין, sabäisch 'Atțar des Himmels, (samâj +. $n$ stat. demonstr.) als עמתר lautet ein kedaritischer Gottesname, in der assyrischen Keilschrift bei Delitzsch, Par. 299 A-tar-sa-ma-a-a-in. Woher weiss Schrader (KAT2 $4 \mathrm{~K}_{4}, \mathrm{I} 3$ ), dass es eine Göttinn ist?

\section{II. רצב, Gold = Ringgold.}

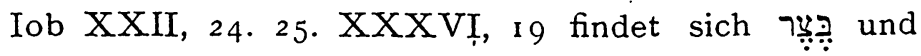
רü „Gold“", und zwar ist XXII, 24 herzustellen: ,Ophirgold und Bachgold." Bekanntlich circulierte in ältester Zeit das rohe Gold in Aegypten und Vorderasien in Ringen von bestimmtem Gewicht, auch kleinen, s. die Litteratur bei Hultsch, Gr. . $R$. $M$. §. 4I, 9. Io. Die Bedeutung Ring und Kreis in dieser 


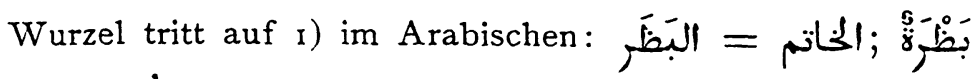
=, der Ring des Siegelrings ohne

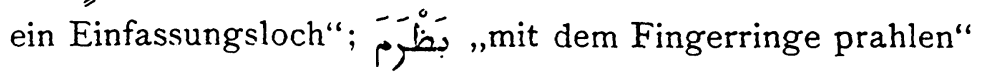
(jamanisch?). Auch البَظظ ,clitoris“" wohl Fleischring um ein Loch, u. s. w.; 2) בטרא, Längenmaass: Fingerbreite ארבעה בטרי ; Fingerumkreis: Levy, Wb. zur Mischnah $=\pi \alpha \lambda \alpha \iota \sigma \tau r_{r}^{\prime}$ bei HuLrsch $\S 5,2$; 3) im Hebräischen Mich. II, I 2 Steinkreis und Mauerkreis = גרדות צאן Num. 32. 16; I Sam. 24, 4; Zeph. 2, 6, zum Schutze teils gegen Unwetter, teils gegen Feinde: מגדל ערר Mi. 4, 8; Gen. 35, 21; damit identisch der Stadtname, wie ברבא, und man darf fragen, ob daher richt auch מְְִָּ und, denominativ,

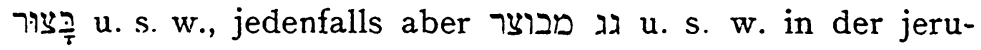
salemischen Mischnah Levy I, 252; 4) die Namen des

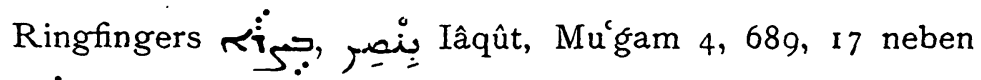
"kleiner Finger" gehören vielleicht trotz Abweichung von der Regel als Lehnwörter hierher.

\section{III. $\Delta \alpha \varrho \varepsilon\llcorner x o ́ s$.}

In der Abhandlung (Fourn. as. VII, IV, p. 479), in welcher Jules OpPert von paras „Hälfte" Kenntniss giebt,

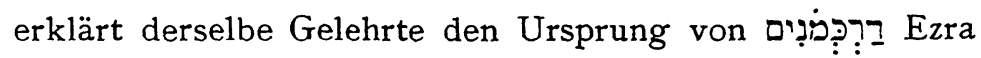
II, 69; Nehem. VII, 70. 7I-72 aus darag mana = "Grad einer Mine" $=\frac{1}{60}$ Mine, sofern darag zunächst $\frac{1}{60}$ Kreisbogen bedeute. ${ }^{\mathrm{I}}$ ) Von allem andern abgesehn, steht aber in diesem Namen im Hebräischen, Syrischen und Griechischen das $==\varkappa$ fest, und ferner bliebe der Wegfall des

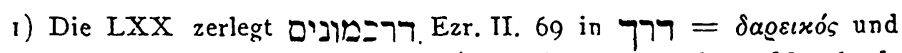

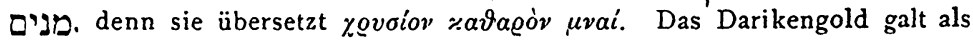
besonders rein: Herod. 4, 166.

Zeitschr. f. Assyriologie, II. 
ררבונות in der Mischnah, und in זמקת unerklärlich. Auch nach Blau's unmöglicher Deutung des Dariken als "Goldstück“ זרד (ZDMG 6, 482) lohnt sich eine neue Erwägung der Sache.

Geht man davon aus, dass das Grundwort persisch ist, so darf man annehmen, dass dasselbe in zwei Gestalten: ררכ und un in die westlichen Sprachen überging. Die letztere wäre das Wort mit der Casusendung am, entweder Nominativ eines Neutrums der $a$-Stämme, oder Accusativ eines Masculinums. Gewöhnlich ist die Herübernahme eines Nominativs, auf $\boldsymbol{w}$, wie in ohne dieses haben wir is gegenüber schen Wortes. Also ka, bezw. kam wäre die persische Endung. Was aber ist ררו? Wer die Geschichte der vorderasiatischen Münzprägung von BRANdis, und Hultsch's Metrologie liest, wird von vornherein geneigt sein, diesen Namen mit dem persischen Grosskönig in Beziehung zu bringen. Gesenius im Thes. 353 und de Lagarde in den Abhandlungen 242, 13 haben dagııxós gradezu gleich

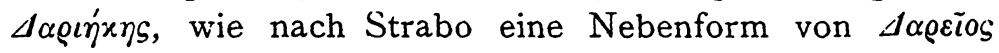
lautete, gesezt. Einen analogen Zusammenhang hat vielleicht 'A @oi\%as, nach Ctesias Name Artaxerxes' II., mit

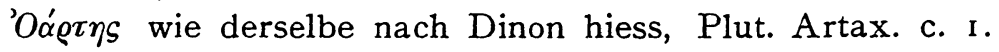
NöLdeke in Encycl. Britannica, 'Persia' p. 576 schreibt Oarses. - Mit $\Delta \alpha \varrho \iota \eta^{\prime} \wedge \eta s$ stimmt in der That - scheinbar, s. unten - die Aussprache des Goldstücks, wie sie Bar Alı 3231 ausdrücklich überliefert, vollständig überein:

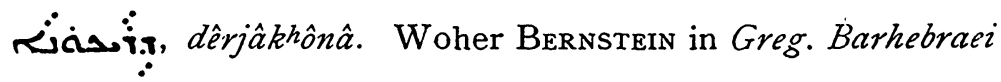
chronici syriaci . . . . specimen I Lips. I 822, p. 49 ff. מحم „dărîjăkîunâ" hat, kann ich nicht nachsehn. Das Thema $\Delta \alpha \varrho \iota \eta$ - hat die meiste Aehnlichkeit mit dem von $\Delta \alpha \varrho \varepsilon \iota \alpha \tilde{\imath}-o s$ Xen. hist. Gr. II, I, 8. - רריוש, schon eine Verkürzung von Dârayava(h)uś (vgl. das babylonische Da-a-ri-ya-avus bei Schrader, ABK 363, Da-(a-)ri-'-vuśs, Da-ri-'-urs 


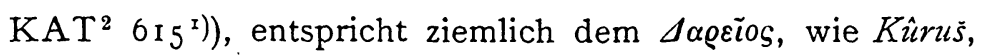
בור dem Kũoos u. s. w. Mit Abwerfung der Flexion erscheint im Pahlawî ראריו (ZDMG 33, I46), später ראראי

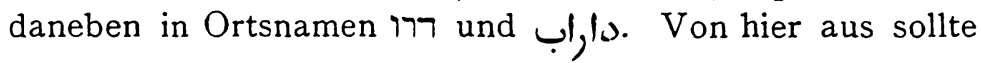
man רריוך Dâraizuak oder Dâraiuk oder a. dgl. erwarten. Die Vocale des armenischen Dareh, dessen $h$ nach DE LAGARDE, $A b h$. 183, I 4 aus dem $k$ entstanden ist, könnten doch nur eine sehr späte Gestalt des Wortes vertreten.

Dieses $k(a)$ würde die Wortendung sein, mit welcher gewisse persische Dialekte die Grundform zu erweitern lieben, analogisch, nicht um Deminutive zu bilden.

Mit dieser $Z$ weisylbigkeit $z$ wischen dem $r$ und $k$, oder wenigstens mit einem consonantischen $j$, lässt sich weder die Schreibung דרב der Bibel und Mischnah, noch die von

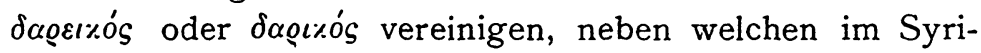
schen auch die Aussprache سiَ drîkhônâ steht; so vocalisiert das nestorianische MS von de Lagarde, Praeter-

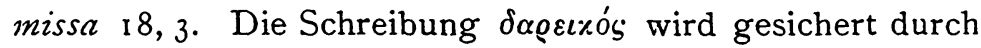
eine Inschrift von Erythrae aus der Zeit des Maussolos (ungefähr 375-351): Dittenberger, Sylloge 84. In einer spartanischen Inschrift von Tegea, welche man für älter als 416 v. Chr. hält, findet sich zweimal $\delta \alpha \varrho \iota(x) o ́ s$ (accus. pl.)

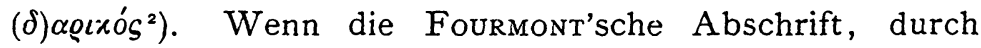
welche jene allein bekannt ist, irgendwie zuverlässig wäre, würde diese Orthographie gegen die Ableitung von Dareios noch schwerer ins Gewicht fallen.

In der Zeit Artaxerxes' I. (?), Nehemia's und Herodot's zeigt sich bestimmter, dass die Keilinschriften eine Orthographie festhalten, welche hinter der gleichzeitigen Aussprache zurückgeblieben war. Auf der ägyptischen Vasen-

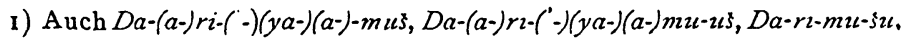

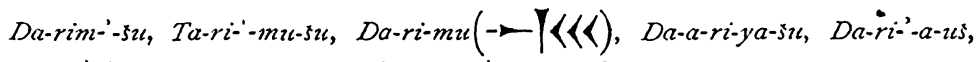
Da-ri-'-(ut-)-su, Da-ra-mus und Da-ar-'su. - Red.

2) Die Litteratur bei HuLrsch, Metrol. p. 485 ist mir nur teilweise zugänglich. 
inschrift bei SPIEGEL, die altpersischen Keilinschriften $188 \mathrm{I}$, p. 68. I 25 wird Artaxerxes I. (?) geschrieben Ardakhcashca, in der babylonischen Version derselben Inschrift nach BEZOLD und Schr ADER (s. diese Zeitschr. I884, p. I I und N. I): Ar̀-ta'-ha-ša-is-su. Schrader (ABK 319; $\mathrm{KAT}^{2} 615$ ) schreibt denselben Namen des zweiten Artaxerxes im babylonischen Texte $A r-t a k-s a t-s u^{\mathrm{I}}$ ), welches mit Metathese das Urbild von ארתחשישת der Bibel wäre. Nöldeke, Encycl. Brit. 'Persia' p. 573 weist auf ägyptische Formen und auf' $A \varrho \tau \alpha \xi^{\xi} \varepsilon \sigma \eta \eta^{\prime}$ einer griechischen Inschrift hin. Man beachte namentlich $d$ für $t$

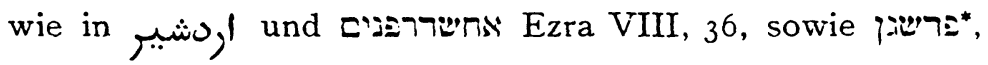

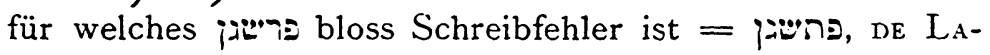
Garde, Armen. Stud. 1838²). Für Katapatuka erscheint

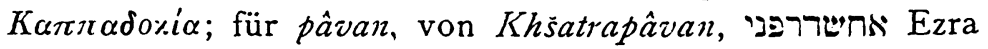
VIII, 36. Freilich zeigt die biblische Orthographie persischer Namen auch sonst Entstellungen: נשיתון wahrscheinlich für נושת gradezu = nipistam, neutr., mit aramäischem

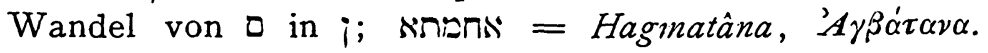

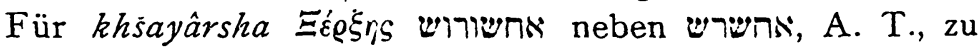
sprechen Ahšôros, sodass $j$ ausgefallen, gegen гiusur, חשיאר: (Lepsius, ÄZ 1877, I27; Schrader, KAT ${ }^{2}$ 615). Bei dieser Sachlage hat man keine Sicherheit dagegen,

I) שת- kann zur Bestätigung der Gleichung khsatra $=-\sigma a r \alpha$ dienen, s. DE LAGarde, $A b h .46$. Denn das letzte $\boldsymbol{U}$ ist vielleicht eine dialektische (medische?) Nominativendung, vgl. SPIEgel p. 172. Mithin wäre Ma@v́aazıs $={ }^{*}$ Parıkhsatr $=\Phi a ́ g \zeta \iota \iota s$ nach Strabo bei LAG., $A b h .183=\Phi a ́ g \sigma \iota \iota s$

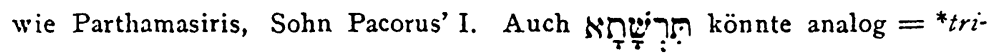

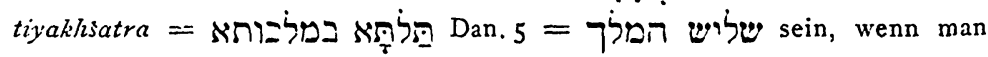
Ausfall von wenigstens $\Pi$ nicht für unmöglich hält.

2) Von بulut, "Antwort" = pat durch die Präposition $\boldsymbol{E} \Omega \Xi$, das $\mathrm{r}$ ä $\mathrm{ml}$ i ch gegenübergestellte entsprechende

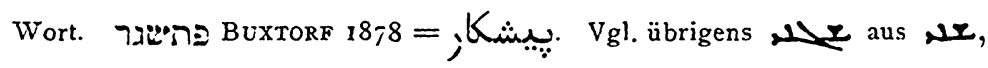

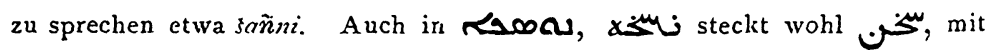
welchem Praefix? 
dass bei ודרבמנים ודר in nicht dennoch ein consonantisches ' oder $"$ zusammengeschmolzen seien, zumal bei der Verlängerung des hebräischen Wortes.

Wir hatten bisher für das Goldstück stillschweigend die Form דריך oder als Doppelgängerin des Namens Darius und als Substantiv vorausgesezt. Allein es ist nicht verständlich, warum der Name Darius in Vorderasien in einer dialektischen Form Darik sollte umgelaufen sein, sobald er die Münze bezeichnete, also grade bei seinem alltäglichsten Gebrauche. Auch den andern Namen der persischen Könige im Munde von Griechen und Semiten fehlt solche vielleicht lokal übliche Endung auf $k$. Dazu scheint mit dieser Aussprache im Widerspruch der Sprach-

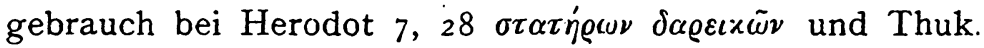

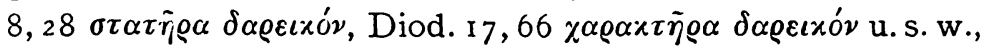
wonach das Wort Adjectiv ist. In der oben erwähnten

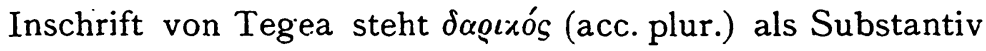
allein. ${ }^{1}$ ) In der That haben die griechischen Grammatiker $\delta \alpha \varrho \varepsilon \iota \%$ S für ein Adjectiv gehalten, und als Zusammenziehung von $\delta \alpha \rho \varepsilon \iota \alpha \times o ́ s$ betrachtet. Allein auch von letzterer Erklärung abgesehn, es scheint im Griechischen keine genau analoge Ableitung eines Adjectivs auf - $\varepsilon \iota o_{s}$ von - $\varepsilon i o s ~ z u$

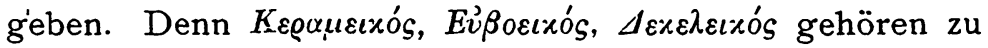

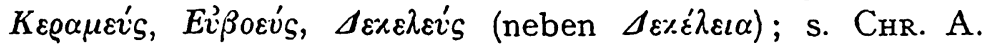
Lobeck. Pathol. Gr. serm. elem. I, 225; II, I39. Diess bestätigt die Annahme, dass das $*$ in $\delta \alpha \varrho \varepsilon \iota \% o ́ s$ vorgriechischen Ursprung hat, ohne auszuschliessen, dass schon Herodot es als griechische Endung auffasste (vgl. IV, I 66 und VII, 28

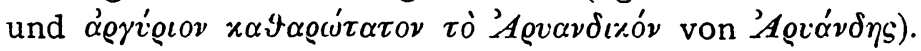

Spricht immerhin schon der adjectivische Gebrauch des Wortes im älteren Griechisch für dieselbe Verwendung des persischen Wortes, so deutet dieselbe auch die Endung i, ôn an, welche an ררב uרבמ angehängt ist. Denn

1) Uebrigens kommt $\delta \alpha \varrho \varepsilon \iota o_{s}$ nur für die Münze vor, nie sonst gleich „dariisch". 
diese ist am ehesten Adjectivendung. Eine Deminutivbildung, noch dazu bei einem nichtpersischen Volke, gliche einem schlechten Scherz und hat die Analogie nicht für sich; vgl. „Darii, Philippi, Fritz, Louis, Napoléon“. Den Singular auf ôn sezt schon das fem. דרבונות der Mischnah voraus, sodass man die Endung nicht als Erweiterung nur

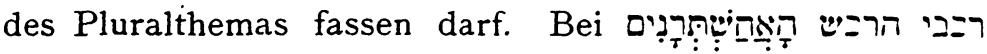
Esth. VIII, 10 „Reichspostreiter" könnte zur Not $\hat{a} n$ schon persischen Ursprungs sein. Vollständig lautete die Verbindung שקל (תקל) ררבמן oder שי אדרבן.

Sobald man nun annimmt, dass jenes persische Adjectiv, welches auf die angegebene Weise gefolgert werden darf, den Sinn von „dariisch" gehabt hat, so vermehren sich noch die Schwierigkeiten, welchen schon die Gleich-

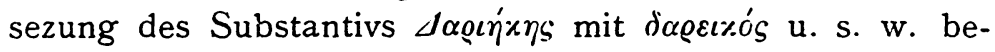
gegnete; denn es ist kaum $z u$ bezweifeln, dass in einem altpersischen Dialekte Patronymica von $\mathrm{P}$ er s o ne n namen nur mittelst der Endung iya gebildet werden konnten, welche um ein secundäres $k a$ vermehrt werden mochte, $=\hat{\imath} k$ im Pahlawî. Auch wurde das Thema, an welches iya trat, höchstens um einen kurzen Vokal verkürzt, vgl. Hakhâmanish-iya und Babiruv-iya; weiter zu kürzen als $D \hat{a}$ rayav-iya, also Dâriaw-ik berechtigt die Analogie nicht. - Für das Patronymicum auf $k$ haben wir ein Zeugniss in Ezra IV, 9

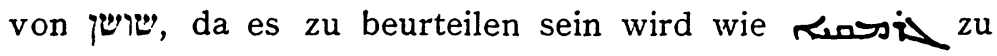

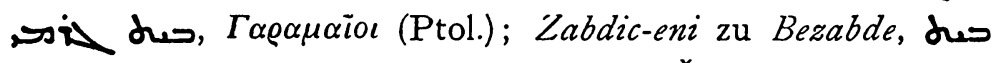
וכז. (Ammian) u. s. w. Selbst wenn Šušinak der elamitischen Inschriften ,susianisch" bedeutet, was OPPERT (den ich nicht nachlesen kann) in Abrede stellt, so ist die Entzifferung wohl noch nicht so sicher, um über die Natur des Vocals im Suffix $-a k,-i k$ (?) etwas zu wissen; s. Schrader, KAT $^{2}$ 6ri. איא Ezra V, 6; VI, 6 wäre ebenso anzusehn, wenn es „Perser“ bedeutete. Aber diess ist zweifelhaft. Es ist vielleicht aus אפרסחבי verdorben und Amts- 
titel, wie sicherlich Ezra IV, 9, wo ich lese „Richter und Gesandte (Commissare)", nach פריםתק Buxr. I83I. I836, فر ستنه ${ }^{1}$ ).

Aber nicht nur bringt auch ein Adjectiv ררי! einer Ableitung des Dariken von Dareios nicht näher, sondern gegen dieselbe scheint auch ein Unterschied der Quantität in der ersten Sylbe zu sprechen. Abgesehn von der im Syrischen ebenfalls überlieferten Aussprache drikhôn $\hat{a}$ führt namentlich das Vorschlagsalef von אררבינם I Chr. 29, 7 ; Ezra VIII, 27, wie in $y_{\gamma}$

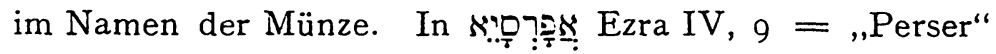
ist das $\$$ ein aus der Umgebung leicht erklärlicher Schreibfehler. Man lese also adrîkônim adrîkemônîm. Die Vocalisationsmechanik der Massora ist durchsichtig genug: sie hat die Vocale von darkemônim auf jene Form übertragen und den Reșt mit Schwa ausgefüllt, gradeso wie sie

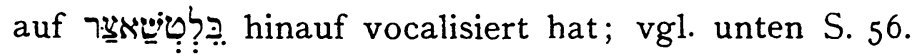

Greifen wir nach diesen Erörterungen noch einmal

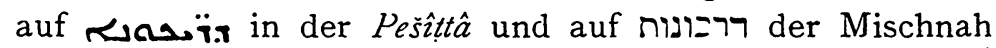
zurück, so ist von vorn herein zur Zeit dieser beiden ein volksthümlicher Gebrauch des Wortes unwahrscheinlich; beide lehnen sich an das A. T. an. Die Mischnah entdeckt darin דרך,,Weg“ (Buxr. 577); die syrischen Uebersetzer haben auch von $\nabla$ ria nichts gewusst, da es vielmehr פרשנן* war. Auch im Pahlawî ist, so viel ich sehe, der

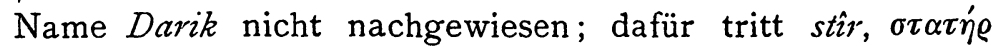
ein (WEST, Uebers. von Shâyast lâa shâyast), wie schon auf dem Löwen von Abydos. Mithin ist bei den Syrern an ein einheimisches, etwa persisches Vorbild der Schulaus.

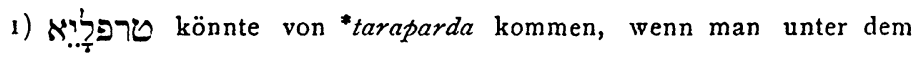
Einfluss des vorangehenden 7 schon damals die Aussprache $d_{0}^{\prime}$ fïr möglich halten will. Es wären die Provincialen ,jenseits der Brücke“ über den Euphrat bei Thapsacus, חספ, d. h. das Wort persisches Gegenstück zu עבר נהריא. Diess passt in den Zusammenhang. 
sprache derjak-ônâ schwerlich zu denken. Vielmehr dürfte

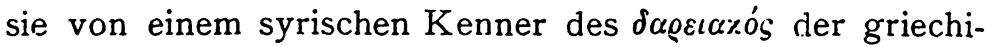
schen Grammatiker herrühren.

Die Schwierigkeiten, welche eine Ableitung des Dariken von Dareios macht, empfehlen die Erwägung der folgenden Vermuthung, nämlich dar-îk $=$ j) von dar, "Thor" d. i. den 'v́øa des Grosskönigs. Es bezeichnete hiernach ררך den Scheqel nach der Währung der königlich persischen Hof- und Reichsmünze im Gegensatz zu ausserpersischen Währungen. Blieb doch auch die Prägung von Goldstücken ein Vorbehalt des Grosskönigs. Damit ist zu vergleichen, dass die assyrische Inschrift der Gewichtslöwen zu beginnen pflegt: Ikallu des N. N. šar mât Asur (vergl. Schrader, ABK 175), „Palast des N. N., Königs von Assyrien". - Einwendungen gegen diese Etymologie, wie der, dass sie statt der altpersischen Form duvarayâ (Dual) den Ausfall des $v$ wie im Pahlawî vorausseze, liesse sich mit dem oben angedeuteten Archaismus der Keilschriftorthographie, oder noch auf andre Weise - Ausfall von Digamma u. s. w. - begegnen.

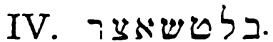

Weder ABK I54, $\mathrm{N}^{\circ} 59^{2}$ noch in $\mathrm{KAT}^{2} 429$ hat SCHRADER diesen Namen aus den Keilinschriften belegt. Somit scheint Balațsu-ușur nur seine Vermuthung zu sein. Einer solchen würde ich die andre entgegenstellen, dass der Name ursprünglich gelautet habe: בלט שר אצר wie נבברראצר, נרגל שר אצר, בל ש]ר] אצר wäre בלט בל באר

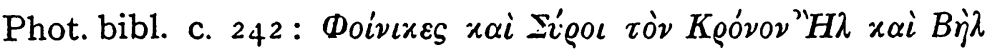

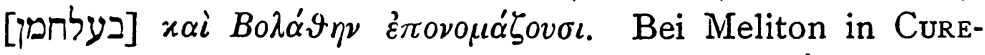
Ton's Spicil. ma, ro kommt eine Aerztin sل d mythologischer, sehr gemischter, Gesellschaft vor. Auch סנבלט könnte denselben Gott mit Sin vereinigen. Die Hergehörigkeit der angeführten Stellen bleibt freilich sehr un- 
sicher, aber doch zu erwägen. Von den aus der Fremde bezogenen Bedeutungen abgesehen ${ }^{\mathrm{I}}$ ), bezeichnet ב 2 im Aram. und Syr.: ,erhaben, beulenartig aus einer Fläche heraustreten, glotzen“, im Arab.: „plötzlich rasch von der eingehaltenen graden Linie abspringen, zucken, sich im Zickzack bewegen", also in beiden mit deutlichem Zusammenhang. Hat damit das assyrische balat "leben" etwas zu thun? Wie?

\section{Balâbâd und al-Muqaijar.}

Weil es den Assyriologen entgangen zu sein scheint, bringe ich in Erinnerung, dass Iâqût, Múgam das $\mathrm{Ba}$ lawat Hormuzd Rassam's بَ schreibt, eine Bildung wie

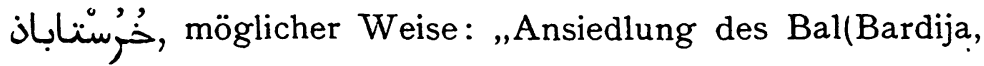
Smerdis)“. Vgl. meine Auszüge aus syr. Akt. pers. Märt. Note 1740.

Al-Muqaijar erwähnt Ibn al-Atîr, Chron. ed. Tornberg, XII, 233, 3 : موضع يعرف بالهقبر [بالهقيّر :1.] وهو ثل كبير بالبطيكهة قرب الغراف. Die Stelle ist· wichtig zur Bestimmung des al-Garrâfkanals.

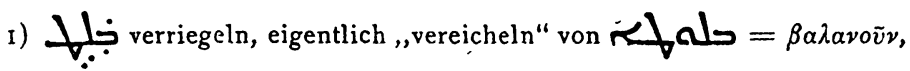

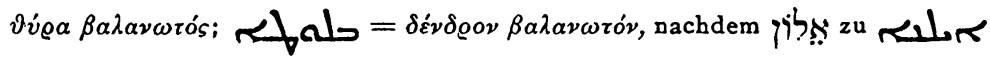
geworden. 